Original

\title{
Validación de la versión en español del cuestionario "Epidemiology of Prolapse and Incontinence Questionnaire-EPIQ"
}

\author{
Montserrat Espuña Pons ${ }^{1}$, Manuel Fillol Crespo², María A. Pascual Amorós ${ }^{3}$, \\ Pablo Rebollo Álvarez ${ }^{4}$, Marta Prieto Soto ${ }^{5}$ \\ ${ }^{1}$ Hospital Clinic i Provincial. Barcelona. ${ }^{2}$ Hospital de la Plana, Castellón. ${ }^{3}$ Hospital Universitario de Canarias, \\ Tenerife. ${ }^{4}$ BAP Health Outcomes, Oviedo. ${ }^{5}$ Astellas Pharma, Madrid, España.
}

\begin{abstract}
Resumen
Introducción: La patología de suelo pélvico afecta a un tercio de la población femenina adulta, por lo que un instrumento para su cribado en práctica clínica habitual sería de gran utilidad. En este estudio se evaluaron las propiedades psicométricas de la versión española del cuestionario estadounidense "Epidemiology of Prolapse and Incontinence Questionnaire-EPIQ" para comprobar su equivalencia al original y poder recomendar su uso en España.

Material y métodos: En el estudio se incluyeron 60 mujeres con problemas de suelo pélvico y 60 pacientes control que respondieron a la versión española del EPIQ. Para validar formalmente el cuestionario se replicó el análisis estadístico utilizado en la validación del original.

Resultados: Factibilidad: 113 mujeres respondieron a todos los ítems del cuestionario y 7 dejaron algún ítem sin contestar. Validez: las dimensiones identificadas fueron: calidad de vida (CV), vejiga hiperactiva (VH), incontinencia anal (IA), dolor y dificultad de vaciado (DDV), disfunción defecatoria (DD), incontinencia urinaria de esfuerzo (IUE) y prolapso pélvico (POP). Se comprobó la validez de contenido comparando las puntuaciones medias de las siete dimensiones entre el grupo de pacientes y el control (prueba $\mathrm{T}$ de Student). Los valores predictivos positivos y negativos del cuestionario fueron: POP=92,3\% y 82,52\%; IUE= 54,34\% y 84,93\%; VH=60,6\% y 84,52\% y IA=24,32\% y 92,68\%. Fiabilidad: el coeficiente alfa de Cronbach global fue 0,94 y para las distintas dimensiones: 0,96 (CV); 0,91 (VH); 0,63 (IA); 0,72 (DDV); 0,75 (DD) y 0,61 (IUE).

Discusión: La versión española del cuestionario EPIQ muestra adecuadas factibilidad, validez y fiabilidad para ser utilizado en la práctica clínica como instrumento de cribado de la patología de suelo pélvico.

Palabras clave: Suelo pélvico. Cuestionarios. Estudios de validación.

\section{Spanish validation of the Epidemiology of Prolapse and Incontinence Questionnaire-EPIQ}

\section{Abstract}

Introduction: One out of three women suffers from pelvic floor disorders. Thus, an instrument to evaluate this pathology in common clinical practice would be very useful. In this study the psychometric characteristics of the Spanish version of the US questionnaire "Epidemiology of Prolapse and Incontinence Questionnaire-EPIQ" have been evaluated, in order to test its equivalence to the original and allow for its use in Spain.

Material and Methods: 60 women with pelvic floor pathology and 60 control women filled in the Spanish version of the EPIQ. The questionnaire was validated following the statistical procedure used in the validation of the original form.

Results: Factibility: 113 women filled in all questionnaire items and 7 leaved some of them blank. Validity: 7 dimensions were indentified: quality of life $(\mathrm{GoL})$, overactive bladder $(\mathrm{OAB})$, anal incontinence (AI), micturition difficulty/pain (MD/P), functional defecation disorders (FDD), stress urinary incontinence (SUI), and pelvic organ prolapse (POP). The validity of contents was proved comparing the mean scores for each dimension in patients and control (T-student test). Positive and negative predictive values were $\mathrm{POP}=92,3 \%$ and $82,52 \%$; $\mathrm{SUI}=54,34 \%$ and $84,93 \%$; $\mathrm{OAB}=60,6 \%$ and $84,52 \%$ and $\mathrm{AI}=24,32 \%$ and $92,68 \%$. Reliability: Global Cronbach's-alfa was 0,94 and for the different dimensions: 0,96 (QoL); 0,91 (OAB); 0,63 (AI); 0,72 (MD/P); 0,75 (FDD) y 0,61 (SUI).

Discussion: The Spanish version of the EPIQ questionnaire presents adequate factibility, validity and reliability to evaluate pelvic floor pathology in clinical practice.

Keywords: Pelvic floor. Questionnaires. Validation studies.

$\mathrm{L}^{\mathrm{a}}$ a patología de Suelo Pélvico es un trastorno que afecta a una tercera parte de la población femenina adulta ${ }^{1,2}$. Estos trastornos raramente se presentan de manera aislada ${ }^{1,3-7}$, por lo que algunos autores consideran que se debería incorporar a la práctica clínica habitual la detección de síntomas del resto de trastornos de suelo pélvico, además de estudiar el correspondiente al motivo de consulta.

Los estudios epidemiológicos consultados sobre prevalencia de patología de suelo pélvico en mujeres, 
muestran cifras muy diferentes. Un estudio llevado a cabo en Estados Unidos ${ }^{3}$, en el que se administró el cuestionario "Epidemiology of Prolapse and Incontinence Questionnaire-EPIQ" a 4.103 mujeres, cifró en un $37 \%$ el porcentaje que sufría una o más alteraciones de suelo pélvico, concretamente, un $15 \%$ padecía incontinencia urinaria de esfuerzo (IUE); un $13 \%$ padecía vejiga hiperactiva (VH); un $6 \%$ tenía prolapso de órganos pélvicos (POP) y, por último, un $25 \%$ sufría incontinencia anal (IA). Otro estudio llevado a cabo también en Estados Unidos ${ }^{8}$, en el que se incluyeron 1.961 mujeres, participantes en la "2005-2006 National Health and Nutrition Examination Survey", encuesta representativa de la población general no institucionalizada, estimó que un $23,7 \%$ sufría una o más alteraciones de suelo pélvico, siendo el desglose de porcentajes los siguientes: un $15,7 \%$ padecía incontinencia urinaria (IU); un $9 \%$ sufría IA y un $2,9 \%$ de mujeres tenía POP. De acuerdo a la revisión sobre el tema llevada a cabo por Lukacz et $\mathrm{al}^{9}$, las cifras de prevalencia de las diferentes patologías del suelo pélvico varían del $1 \%$ al $47 \%$ para la IU, de menos del $1 \%$ al $39 \%$, para la IA y del $16 \%$ al $46 \%$, para el POP. Estas variaciones son debidas, en gran parte, a los diferentes métodos empleados para su diagnóstico, entre otros, cuestionarios no validados formalmente ${ }^{9}$; a las diferentes definiciones utilizadas y a las características de la muestra de la población estudiada.

La patología del suelo pélvico puede ser evaluada a través del diagnóstico clínico, basado en la historia clínica de la paciente. Sin embargo, hay quien defiende que este diagnóstico puede variar según el médico que evalúa a la paciente ${ }^{10}$. Asimismo, hay que tener en cuenta que las pacientes se muestran reacias a consultar al clínico acerca de un tema tan delicado como puede ser la incontinencia urinaria o anal. Por ello, otra forma más válida de medir la presencia y severidad de patología de suelo pélvico consistiría en la utilización de un instrumento en forma de cuestionario auto-contestado, de sencillo uso y formalmente validado. Un instrumento con estas características permitiría realizar una estimación más exacta de la prevalencia de patología de suelo pélvico, ante las diferentes cifras obtenidas en estudios llevados a cabo en diversos países en los últimos años, y permitiría también establecer comparaciones entre diferentes estudios. Por ello, Lukacz et al, desarrollaron y validaron recientemente, en Estados Unidos, el cuestionario "Epidemiology of
Prolapse and Incontinence Questionnaire-EPIQ"9. Este cuestionario consta de 53 preguntas, en parte tomadas de otros instrumentos previamente validados $\mathrm{y}$, en parte, desarrolladas específicamente para el cuestionario. Consta de los siguientes apartados: datos generales e historia ginecológica (ítems 1-11); datos de salud general (items 12-20); preguntas sobre el síndrome VH e IU (ítems 21-27, 31-34); preguntas de afectación de la CV (ítem 30); pregunta sobre prolapso genital (items 35-38); preguntas de incontinencia anal (items 42-47); preguntas sobre dolor y dificultad de vaciado (ítems 28-29); preguntas sobre disfunción defecatoria (ítems 39-41); preguntas sobre vida sexual (items 48-49) y datos sociodemográficos (items 50-53). De las 53 preguntas, las 22 que hacen referencia al impacto en la vida causado por los problemas de suelo pélvico forman la puntuación del cuestionario. Éstas se agrupan en siete dimensiones: Vejiga Hiperactiva; Incontinencia Urinaria de Esfuerzo; Calidad de Vida; Prolapso Pélvico; Incontinencia Anal; Dolor y Dificultad de Vaciado y Disfunción Defecatoria.

El objetivo del presente estudio fue adaptar culturalmente en España el cuestionario "Epidemiology of Prolapse and Incontinence Questionnaire-EPIQ" y comprobar que el cumplimiento de las propiedades psicométricas (factibilidad, validez y fiabilidad) de la versión española adaptada es similar al de la versión original americana, para poder recomendar su uso en la práctica clínica habitual.

\section{MATERIAL Y MÉTODOS}

En primer lugar, se llevó a cabo la adaptación cultural del cuestionario original desarrollado en Estados Unidos para tener la certeza de que la versión española generada es equivalente conceptual y semánticamente a la versión original. En el proceso de adaptación cultural inicialmente se realizó la traducción y retro-traducción del cuestionario, tarea encomendada a dos expertos traductores nativos (británico y español) que, junto con tres miembros del equipo investigador especializados en la validación psicométrica de estas herramientas, consensuaron una versión del cuestionario en Español que, posteriormente fue sometida a al juicio de expertos y pacientes. Así, se llevó a cabo un panel de expertos, con la colaboración de tres médicos especialistas con amplia experiencia en el campo de la uroginecología, de modo que, cada especialista evaluó la pertinencia e importancia de los ítems y 
áreas enunciados. Asimismo, el panel de pacientes consistió en la evaluación de los ítems y áreas enunciados por parte de 21 pacientes ( 7 por especialista participante en el panel) que acudieron por cualquier motivo a consulta de ginecología general. Posteriormente, se pusieron en común las evaluaciones del cuestionario realizadas por cada médico y sus 7 pacientes correspondientes. A partir de las conclusiones derivadas de este debate, se redactó la versión piloto del cuestionario EPIQ.

Una vez realizada la adaptación cultural del cuestionario original, se procedió a evaluar las propiedades psicométricas del cuestionario en una muestra de 120 mujeres. Para ello se incluyeron consecutivamente a 60 mujeres que acudieron por problemas de suelo pélvico a las consultas ginecológicas especializadas en patología de suelo pélvico del IGCON del Hospital Clínic de Barcelona, del Hospital de la Plana de Castellón y del Hospital Universitario de Canarias de Tenerife. Asimismo, se incluyeron consecutivamente 60 mujeres sin patología de suelo pélvico que acudieron a consulta general de ginecología de los citados centros.

Tras prestar consentimiento informado por escrito, estas 120 mujeres contestaron al cuestionario a examen, la versión española del "Epidemiology of Prolapse and Incontinence Questionnaire-EPIQ” (versión completa del cuestionario en el pdf de la página web: www.actasurologicas.info), siguiendo una entrevista estructurada que realizó personal especialmente entrenado a tal fin. El médico responsable en cada caso cumplimentó un sencillo registro de variables que incluía el motivo de consulta, el resultado de la exploración pélvica y la impresión diagnóstica clínica de acuerdo a las definiciones utilizadas en la validación de la versión original americana (Tabla 1).

Además, se obtuvo una segunda respuesta al cuestionario EPIQ a los 15 días de la primera, y en situación clínica estable, en 21 mujeres de la muestra sana de validación y en 24 mujeres de la muestra del estudio.

\section{ANÁLISIS ESTADÍSTICO}

Para validar formalmente la versión española del EPIQ se replicó el análisis estadístico utilizado en la validación de la versión original americana, que siguió los procedimientos estadísticos adecuados para este tipo de estudios.

Para la evaluación de la factibilidad, se analizaron los ítems de manera individual obteniendo los
Tabla 1. Definiciones de la impresión diagnóstica clínica (Lukacz et al, 2005)

\section{Prolapso de órganos pélvicos}

Síntomas de presión, bulto o protrusión y con evidencia en la exploración física de un prolapso de pared vaginal anterior y/o vaginal posterior y/o central de segundo grado o mayor.

\section{Incontinencia Urinaria de Esfuerzo}

Sintoma de pérdida involuntaria de orina con la tos, estornudo o esfuerzo una o más veces al mes y que esta pérdida incomode lo suficiente para motivar la búsqueda de tratamiento y con un test de esfuerzo positivo en el examen físico.

\section{Vejiga Hiperactiva}

Urgencia miccional y/o Incontinencia Urinaria de Urgencia.

\section{Urgencia miccional}

Síntomas de urgencia miccional 1 ó más veces al mes y que le supongan a la paciente molestia suficiente como para buscar tratamiento. Con análisis de orina, mediante una tira reactiva que haya descartado infección y hematuria.

\section{Incontinencia Urinaria de Urgencia}

Síntoma de pérdida involuntaria de orina asociada a urgencia miccional 1 ó más veces al mes y que le supongan a la paciente molestia suficiente como para buscar tratamiento. Con análisis de orina, mediante una tira reactiva que haya descartado infección y hematuria.

\section{Incontinencia anal}

Síntomas de incontinencia de gases o de heces.

\section{Incontinencia de gases}

Pérdida involuntaria de gases una o más veces al mes y que cause suficiente molestia e incomodidad como para buscar tratamiento.

\section{Incontinencia de heces}

Pérdida involuntaria de heces sólidas o líquidas una o más veces al mes y que cause suficiente molestia e incomodidad como para buscar tratamiento.

porcentajes de no respuesta. Además, se analizó el porcentaje de pacientes que no contestaron a alguno de los ítems. Asimismo, se analizaron los efectos techo y suelo medidos como el porcentaje de sujetos con la mejor y peor puntuación posible (0 mejor puntuación posible y 10 peor puntuación posible).

Se evaluó la validez de constructo mediante un Análisis Factorial de Componentes Principales (con rotación Varimax) para identificar las dimensiones del cuestionario y comprobar que replican la estructura original del mismo.

Se realizó un análisis de la consistencia interna mediante el cálculo del coeficiente alfa de Cronbach para el total de los ítems y para cada una de las dimensiones. Además se analizó la fiabilidad testretest mediante el cálculo del coeficiente de correlación intraclase. 
Se evaluó la validez de contenido mediante la comparación de las puntuaciones medias de las siete dimensiones entre los dos grupos de mujeres estudiadas: con y sin patología de suelo pélvico. Para ello se utilizó la prueba t de Student, asumiendo varianzas desiguales.

Se evaluó la validez de criterio mediante la comparación de los diagnósticos proporcionados por el cuestionario EPIQ, con el diagnóstico clínico. Para ello, se calculó la sensibilidad, especificidad, valores predictivos positivos y valores predictivos negativos del cuestionario a examen. Se determinaron los distintos puntos de corte de las dimensiones POP, IUE, VH e IA, según sus deciles y se escogieron aquellos que maximizaban los valores predictivos positivos.

\section{RESULTADOS}

Evaluación de la factibilidad: Se obtuvo un porcentaje de "no respuesta" muy bajo en todos los ítems; la tasa de no respuesta máxima obtenida fue de 1,67\% (2 de 120) en los ítems “¿Cuánto le molestan las pequeñas pérdidas de orina (gotas)?” y
“¿Cuánto le molesta la sensación de tener un bulto en la vagina o de que hay algo que sale de ella?”. Asimismo, 113 mujeres respondieron a todos los ítems del cuestionario, mientras que sólo 7 pacientes dejaron algún ítem sin contestar.

Como se observa en la Tabla 2, se detectó un efecto suelo muy pequeño en algunos ítems, oscilando el porcentaje de sujetos con la peor puntuación posible (10 puntos) entre el 2,54 y el 19,17\%. Asimismo, se observó un claro efecto techo, con un porcentaje de sujetos con la mejor puntuación posible (0 puntos) de entre el 42,50 y el 96,61\%.

Evaluación de la validez de constructo: Las siete dimensiones identificadas en el Análisis Factorial fueron las siguientes (en orden de importancia): Calidad de Vida (CV), que explica el 46,3\% de la varianza total; Vejiga Hiperactiva (VH) (9,01\%); Incontinencia Anal (IA) (6,50\%); Dolor y Dificultad de Vaciado (DDV) (5,55\%); Disfunción defecatoria (DD) $(5,17 \%)$; Incontinencia Urinaria de Esfuerzo (IUE) $(4,57 \%)$ y Prolapso Pélvico (POP) (3,19\%); siendo el $80,32 \%$ el porcentaje de la varianza explicado

Tabla 2. Efectos suelo y techo

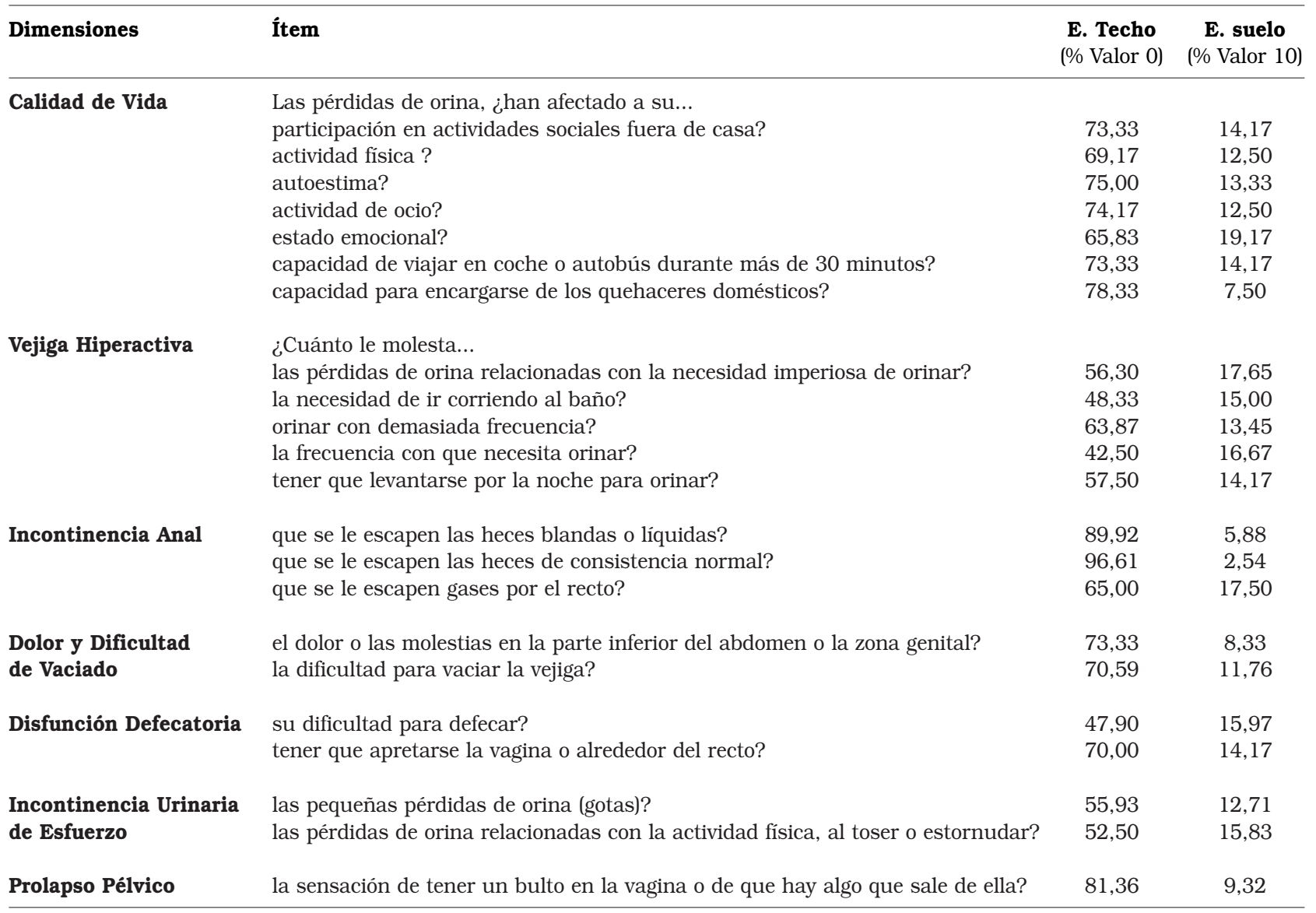


por las siete dimensiones (Tabla 3). Estas dimensiones son las mismas que se identificaron en la versión original del cuestionario.

Evaluación de la fiabilidad: se evaluó la consistencia interna y la fiabilidad test-retest. El indicador calculado para evaluar la consistencia interna del cuestionario es el coeficiente alfa de Cronbach (Tabla 3). Este coeficiente tomó el valor 0,94 para el total de los ítems frente al 0,91 obtenido en la versión original. El coeficiente alfa calculado para las siete dimensiones tomó los siguientes valores: 0,96 (CV); 0,91 (VH); 0,63 (IA); 0,72 (DDV); 0,75 (DD) y 0,61 (IUE). La dimensión PP tiene sólo un ítem por lo que no tiene sentido calcular este coeficiente. Respecto a la fiabilidad test-retest, los valores obtenidos para los coeficientes de correlación intraclase, tanto para los 22 ítems individuales como para las
7 dimensiones, estuvieron comprendidos entre 0,50 y 0,96 , siendo una excepción la correlación obtenida para el ítem “¿Cuánto le molestan las pequeñas pérdidas de orina (gotas)?”, con un coeficiente muy bajo de 0,09.

Evaluación de la validez de contenido: Se identificaron diferencias estadísticamente significativas, al 95\% de nivel de confianza $(\mathrm{p}<0,05)$, entre las mujeres con y sin patología de suelo pélvico, en todas los dimensiones del cuestionario (Tabla 4).

Evaluación de la validez de criterio: Para el cálculo de la especificidad, sensibilidad del cuestionario, valores predictivos positivos y valores predictivos negativos del cuestionario EPIQ, se determinaron los puntos de corte de las dimensiones POP, IUE, VH e IA que maximizan los valores predictivos positivos. Los resultados se muestran en la Tabla 5.

Tabla 3. Análisis Factorial (matriz de componentes rotados) y Fiabilidad

\begin{tabular}{|c|c|c|c|c|c|c|c|c|}
\hline Ítem & $\mathbf{C V}$ & VH & IA & DDV & DD & IUE & POP & Total \\
\hline \multicolumn{9}{|l|}{ Las pérdidas de orina, ¿han afectado a su... } \\
\hline Participación en actividades sociales fuera de casa? & 0,92 & & & & & & & \\
\hline Actividad física? & 0,86 & & & & & & & \\
\hline Autoestima? & 0,86 & & & & & & & \\
\hline Actividad de ocio? & 0,85 & & & & & & & \\
\hline Estado emocional? & 0,79 & & & & & & & \\
\hline Capacidad de viajar en coche o autobús durante más de 30 minutos? & 0,73 & & & & & & & \\
\hline Capacidad para encargarse de los quehaceres domésticos? & 0,70 & & & & & & & \\
\hline \multicolumn{9}{|l|}{ ¿Cuánto le molesta... } \\
\hline Las pérdidas de orina relacionadas con la necesidad imperiosa de orinar? & & 0,75 & & & & & & \\
\hline La necesidad de ir corriendo al baño? & & 0,75 & & & & & & \\
\hline Orinar con demasiada frecuencia? & & 0,67 & & & & & & \\
\hline La frecuencia con que necesita orinar? & & 0,64 & & & & & & \\
\hline Tener que levantarse por la noche para orinar? & & 0,62 & & & & & & \\
\hline Que se le escapen las heces blandas o líquidas? & & & 0,86 & & & & & \\
\hline Que se le escapen las heces de consistencia normal? & & & 0,82 & & & & & \\
\hline Que se le escapen gases por el recto? & & & 0,59 & & & & & \\
\hline El dolor o las molestias en la parte inferior del abdomen o la zona genital? & & & & 0,64 & & & & \\
\hline La dificultad para vaciar la vejiga? & & & & 0,58 & & & & \\
\hline Su dificultad para defecar? & & & & & 0,89 & & & \\
\hline Tener que apretarse la vagina o alrededor del recto? & & & & & 0,79 & & & \\
\hline Las pequeñas pérdidas de orina (gotas)? & & & & & & 0,91 & & \\
\hline Las pérdidas de orina relacionadas con la actividad física, al toser o estornudar? & & & & & & 0,49 & & \\
\hline La sensación de tener un bulto en la vagina o de que hay algo que sale de ella? & & & & & & & 0,81 & \\
\hline Coeficiente alfa de Cronbach & 0,96 & 0,91 & 0,63 & 0,72 & 0,75 & 0,61 & * & 0,94 \\
\hline
\end{tabular}

*La dimensión PP tiene sólo un ítem por lo que no tiene sentido calcular este coeficiente

CV: Calidad de Vida; VH: Vejiga Hiperactiva; IA: Incontinencia Anal; DDV: dolor y dificultad de Vaciado; DD: Disfunción Defecatoria; IUE: Incontinencia Urinaria de Esfuerzo y POP: prolapso pélvico. 
Tabla 4. Puntuaciones medias en las siete dimensiones por grupo de paciente: con y sin patología de suelo pélvico

\begin{tabular}{|c|c|c|c|c|c|}
\hline Dimensión & Grupo de pacientes & $\mathbf{n}$ & Media & Desv. estándar & Valor de $p$ \\
\hline \multirow[t]{2}{*}{$\mathrm{CV}$} & Con patología & 60 & 35,85 & 37,85 & $<0,0001$ \\
\hline & Sin patología & 60 & 3,70 & 12,82 & \\
\hline \multirow[t]{2}{*}{ VH } & Con patología & 60 & 54,87 & 34,47 & $<0,0001$ \\
\hline & Sin patología & 58 & 11,30 & 16,86 & \\
\hline \multirow[t]{2}{*}{ IA } & Con patología & 59 & 17,54 & 28,38 & 0,0423 \\
\hline & Sin patología & 58 & 8,85 & 15,51 & \\
\hline \multirow[t]{2}{*}{ DDV } & Con patología & 60 & 35,08 & 37,35 & $<0,0001$ \\
\hline & Sin patología & 59 & 8,81 & 20,39 & \\
\hline \multirow[t]{2}{*}{ DD } & Con patología & 59 & 39,07 & 39,54 & 0,0059 \\
\hline & Sin patología & 60 & 21,13 & 29,34 & \\
\hline \multirow[t]{2}{*}{ IUE } & Con patología & 60 & 46,00 & 34,05 & $<0,0001$ \\
\hline & Sin patología & 58 & 19,57 & 28,95 & \\
\hline \multirow[t]{2}{*}{ PP } & Con patología & 58 & 28,45 & 42,26 & $<0,0001$ \\
\hline & Sin patología & 60 & 1,67 & 90,51 & \\
\hline
\end{tabular}

CV: Calidad de Vida; VH: Vejiga Hiperactiva; IA: Incontinencia Anal; DDV: dolor y dificultad de Vaciado; DD: Disfunción Defecatoria; IUE: Incontinencia Urinaria de Esfuerzo y POP: prolapso pélvico.

Tabla 5. Valores de sensibilidad, especificidad y valores predictivo positivo y negativo de las dimensiones del cuestionario EPIQ respecto del diagnóstico clínico

\begin{tabular}{lcccc}
\hline & POP & IUE & VH & IA \\
\hline Sensibilidad & 40 & 69,44 & 60,6 & 60 \\
Especificidad & 98,83 & 74,69 & 84,52 & 73,07 \\
VPP & 92,3 & 54,34 & 60,6 & 24,32 \\
VPN & 82,52 & 84,93 & 84,52 & 92,68 \\
VP & 10,34 & 21,01 & 17,09 & 7,56 \\
VP+FN & 25,86 & 30,25 & 28,21 & 12,61 \\
\hline
\end{tabular}

\section{DISCUSIŌN}

En el estudio que aquí se presenta se realizó la adaptación cultural y validación en España del cuestionario EPIQ, para ser empleado en la práctica clínica como instrumento de cribado de la patología de suelo pélvico y en investigación epidemiológica. . La versión española es equivalente, semánticamente, a la original en inglés. La nueva versión española del cuestionario EPIQ mostró adecuadas factibilidad, fiabilidad, validez de constructo, de contenido y de criterio.

El EPIQ es uno de los primeros cuestionarios desarrollados con metodología rigurosa, que permite estudiar poblaciones de mujeres con riesgo de patología del suelo pélvico, para determinar la presencia o no de una serie de problemas de salud que se pueden englobar dentro de este concepto y a su vez evaluar la afectación que produce su presencia en la calidad de vida de las mujeres que las padecen. Su utilización en investigación epidemiológica en mujeres de la población general, ha permitido tener una visión global de la patología del suelo pélvico y de los problemas más frecuentes y sus asociaciones ${ }^{3}$. El EPIQ puede ser utilizado también en la práctica clínica como modelo de historia clínica y como cuestionario de sintomas, ya que contiene apartados de datos generales e historia ginecológica; datos de salud general y preguntas sobre Vejiga Hiperactiva; Incontinencia Urinaria de Esfuerzo; Incontinencia Urinaria de Urgencia, Dificultad de Vaciado vesical, Prolapso genital; Incontinencia Anal;Disfunción Defecatoria, Dolor pélvico y actividad y función sexual

Es un cuestionario fácil de cumplimentar ya sea de forma auto-administrada o con ayuda de un entrevistador. En nuestro estudio se ha comprobado la adecuada factibilidad de la versión española del cuestionario analizando los ítems de manera individual y observando una tasa de "no respuesta" muy baja en todos los casos. La tasa de no respuesta máxima fue inferior al $2 \%$ y observada sólo en 2 de los ítems. Además, el porcentaje de pacientes que no contestaron a algunos de los items fue muy bajo. Por tanto la versión española del cuestionario EPIQ es fácilmente comprendida por las pacientes a las que está dirigido que pueden auto-cumplimentarlo sin problemas. 
Las mujeres con patología del suelo pélvico refieren aparte del síntoma en si mismo, un grado de molestia en su vida, que puede hacer que, frente a un mismo problema la decisión de tratamiento sea distinta. El EPIQ permite con su escala de molestia en cada uno de los sintomas principales, distinguir entre las mujeres que tienen un determinado síntoma, pero no les molesta, de las que el mismo síntoma les supone una grave molestia.

Para estas escalas de molestia se observó la existencia de un efecto suelo muy pequeño (dificultad en discriminar entre los peores estados de salud según el cuestionario; porcentaje de pacientes con la peor puntuación posible) en algunos ítems que en ningún caso superó el 20\%. Además se detectó efecto techo (dificultad en discriminar entre los mejores estados de salud según el cuestionario; porcentaje de pacientes con la mejor puntuación posible), tal y como se esperaba, dado que se trata de un test de detección de patología, que no se presenta en un elevado número de pacientes (la incontinencia anal, por ejemplo, es poco frecuente). En este tipo de cuestionarios, el efecto techo es similar al del presente instrumento.

Para evaluar la validez de constructo se realizó un Análisis Factorial, a través del cual se identificaron siete dimensiones (en orden de importancia): CV; VH; IA; DDV; DD; IUE y PP. Estas dimensiones son las mismas que se identificaron en la versión original del cuestionario. El coeficiente alfa de Cronbach de la versión española del cuestionario fue superior a 0,80 y similar al de la versión original. En cuanto a los coeficientes alfa calculados para las siete dimensiones, en algunos casos $(\mathrm{CV}$, $\mathrm{VH}$, IA e IUE) fueron incluso superiores a los obtenidos en el cuestionario original. Respecto a la reproducibilidad test-retest, el coeficiente de correlación intraclase puede ser calificado de moderadoalto, siendo una excepción el coeficiente del ítem “¿Cuánto le molestan las pequeñas pérdidas de orina (gotas)?”, con un coeficiente muy bajo de 0,09.

Se evaluó la validez de contenido de la versión adaptada, confirmando la hipótesis planteada al inicio del estudio al comprobar que las puntuaciones medias de las pacientes sin patología de suelo pélvico, en cada una de las siete dimensiones, son menores que las puntuaciones medias de las mujeres con dicha patología, siendo estas diferencias estadísticamente significativas al 95\% del nivel de confianza.

En la validación de la versión inglesa del cuestionario, se determinaron unos criterios de diagnós- tico clínico que, cuando la patología lo permite, combinan los sintomas con la observación realizada en la exploración física (por ejemplo: IUE cuando la prueba de esfuerzo es positiva, prolapso genital cuando se observa el descenso de la pared vaginal que supera el introito vaginal). Se observó que las mujeres en las que el médico había realizado un diagnóstico clínico concreto, realizado sin conocer los datos del cuestionario que había llenado la mujer, el grado de afectación referido por la mujer en el cuestionario, era significativamente superior que las que no tenían el diagnóstico clínico de esta patología. Esto tenía poder suficiente como para poder determinar un punto de corte específico en las escalas de afectación para poder optimizar el valor predictivo de cada patología ${ }^{9}$. Estos mismos diagnósticos clínicos se han utilizado en la validación de la versión española del EPIQ.

Respecto a la evaluación de la validez de criterio, los valores obtenidos de sensibilidad, especificidad y valores predictivos positivo y negativo del cuestionario EPIQ respecto del diagnóstico clínico, son un poco más bajos que los de la versión original americana. Esto puede ser debido a que la muestra utilizada en la validación del cuestionario original contenía un mayor número de diagnósticos positivos, sobre todo para Incontinencia Anal (35\% vs 12\% en el presente estudio). Por otro lado, en el presente estudio han participado tres especialistas pertenecientes a tres centros diferentes, aunque utilizando las mismas definiciones para realizar el diagnóstico clínico, mientras que en la validación del cuestionario original, participaron dos especialistas de un mismo centro, utilizando las mismas definiciones clínicas.

En resumen el cuestionario EPIQ, en el contexto de mujeres con patología del suelo pélvico, se situaría no como un instrumento diagnóstico, sino un instrumento que permite hacer un cribado global de toda la patología del suelo pélvico en su conjunto. Una vez detectadas las distintas patologías, se debería proceder a la confirmación diagnóstica, ya sea mediante diagnóstico clínico o con pruebas complementarias. En la práctica clínica si lo que se pretende es sólo saber si existe o no un determinado problema, el EPIQ pude ser suficiente. En investigación de resultados, se ha de completar con cuestionarios específicos de cada patología, que evalúen no solo la existencia o no del problema, sino que puedan utilizarse como escalas para analizar los resultados de los tratamientos aplicados. 
Se puede concluir que la versión española del cuestionario EPIQ muestra adecuadas factibilidad, validez y fiabilidad para ser utilizado en la práctica clínica como instrumento de cribado de la patología de suelo pélvico.

\section{Agradecimientos}

Este estudio ha sido financiado con la ayuda de Astellas-Pharma S.A.

\section{REFERENCIAS}

1. Davis K, Kumar D. Pelvic floor dysfunction: a conceptual framework for collaborative patient-centred care. J Adv Nurs. 2003;43 (6):555-568.

2. Klingele CJ. Advances in urogynecology. Int J Fertil Womens Med. 2005;50(1):18-23.

3. Lawrence JM, Lukacz ES, Nager CW, Hsu JW, Luber KM. Prevalence and co-occurrence of pelvic floor disorders in communitydwelling women. Obstet Gynecol. 2008;111(3):678-685.

4. Buchsbaum GM. Urinary incontinence and pelvic organ prolapse. Minerva Urol Nefrol. 2006;58(4):311-319.

5. Lacima G, Espuña M. Pelvic floor disorders. Gastroenterol Hepatol. 2008;31(9):587-595.
6. Villet R. Case management of pelvic floor disorders. Bull Acad Natl Med. 2005;189(7):1541-1558.

7. Lacima G, Pera M. Combined fecal and urinary incontinence: an update. Curr Opin Obstet Gynecol. 2003;15(5):405-410.

8. Nygaard I, Barber MD, Burgio KL, Kenton K, Meikle S, Schaffer $\mathrm{J}$, et al. Pelvic Floor Disorders Network. Prevalence of symptomatic pelvic floor disorders in US women. JAMA. 2008;300(11): 1311-1316.

9. Lukacz ES, Lawrence JM, Buckwalter JG, Burchette RJ, Nager CW, Luber KM. Epidemiology of prolapse and incontinence questionnaire: validation of a new epidemiologic survey. Int Urogynecol J. 2005; 16(4):272-284.

10. Barber MD. Questionnaires for women with pelvic floor disorders. Int Urogynecol J Pelvic Floor Dysfunct. 2007;18(4):461465.

Correspondencia autor: Dra. Montserrat Espuña Pons CGON. Hospital Clinic i Provincial.

Villarroel 170 - 08036 Barcelona

Tel.: 932275400

E-mail autor: 12922mep@comb.es

Información artículo: Original - Incontinencia femenina

Trabajo recibido: febrero 2009

Trabajo aceptado: marzo 2009 